Vietnam Journal of Mechanics, VAST, Vol.40, No. 1 (2018), pp. $33-45$

DOI:10.15625/0866-7136/9840

\title{
NONLINEAR DYNAMIC RESPONSE OF ES-FGM PLATE UNDER BLAST LOAD
}

\author{
Pham Hong Cong ${ }^{1}$, Nguyen Dinh Duc ${ }^{2, *}$ \\ ${ }^{1}$ Centre for Informatics and Computing, VAST, 18 Hoang Quoc Viet, Hanoi, Vietnam \\ ${ }^{2}$ VNU University of Engineering and Technology, Hanoi, Vietnam \\ *E-mail: ducnd@vnu.edu.vn \\ Received May 24, 2017
}

\begin{abstract}
The paper studies nonlinear dynamic response of an eccentrically stiffened FGM (ES-FGM) plates resting on the elastic foundations subjected to blast and thermal loads with temperature dependent material properties using the Reddy's higher order shear deformation plate theory, stress function, the Galerkin and the fourth order Runge-Kutta methods. The plates are reinforced by outside stiffeners and both stiffeners and plate are deformed under temperature. The effects of material and geometrical properties, temperature-dependent material properties, elastic foundations and stiffeners on the nonlinear dynamic response of the ES FGM plate under blast and thermal loads in thermal environments are studied and discussed.
\end{abstract}

Keywords: Functionally graded materials, nonlinear dynamic response, Reddy's third order shear deformation plate theory (TSDT), blast load.

\section{INTRODUCTION}

In the mid-1980s, functionally graded material (FGM) was introduced for the first time. FGM is characterized by spatial variation in material properties as it is composed of at least two different components with a gradual changing of volume fraction along at least one direction. The purpose of this concept is to combine the best properties of two or more constituents. Additionally, it helps to eliminate the interface problems commonly found in composite materials in order to achieve a smoother stress distribution.

In recent years, many important studies have been researched about vibration and nonlinear dynamic response of an FGM plate and shell. In the vibration and nonlinear dynamic response of FGM research field, Duc et al. [1]; Dung and Nga [2] using the FSDT presented the nonlinear analysis of stability for ES-FGM plate under mechanical and thermal loads; Duc [3] studied the nonlinear dynamic response of FGM plate and shell; Duc et al. $[4,5]$ investigated the nonlinear vibration and dynamic response of ES-FGM plate using FSDT [4], TSDT [5]; Alijani and Amabili [6]; Ready and Chin [7]; Ungbhakorn and Wattanasakulpong [8] considered the FGM plate without stiffeners.

(C) 2018 Vietnam Academy of Science and Technology 
Vibration of cylindrical shell has been mentioned in some studies. Thang et al. [9] investigated the thermo mechanical buckling and post-buckling of cylindrical shell with functionally graded coatings and reinforced by stringers; Dung and Nam [10] did research on circular cylindrical. Duc et al. [11] studied the nonlinear dynamic and vibration of the S-FGM shallow spherical shells resting on elastic foundations including temperature effects.

The study about double curved shallow shell has been found in the works of Bich et al. [12]; Duc [13]; Bich et al. [14].

In the vibration of plate and shell subjected to blast load research field, Hau et al. [15], studied the dynamic response of laminated composite plate subjected to blast loading using CS-FEM-DSG3; Duc and Cong [16] investigated the nonlinear dynamic response and vibration of sandwich composite plates with negative Poisson's ratio in auxetic honeycombs; Nelson Lam et al. [17]; Duc et al. [18] presented the nonlinear dynamic response of FGM plate using TSDT (without stiffeners). Duong and Duc [19] considered the evaluation of elastic properties and thermal expansion coefficient of composites reinforced by randomly distributed spherical particles with negative Poisson's ratios.

This paper presents analytical solutions for the nonlinear dynamic response of the shear deformable ES-FGM plates on elastic foundations using both of the Reddy's third order shear deformation plate theory (TSDT), stress function, the Galerkin method and the fourth order Runge-Kutta method. The work also shows the influences of geometrical parameters, the material properties, the elastic foundations, eccentric stiffeners, temperature on the nonlinear dynamic response of the ES-FGM plates under blast and thermal loads.

\section{PROBLEM FORMULATION}

\subsection{Model of an eccentrically stiffened FGM (ES-FGM) plate}

An eccentrically stiffened functionally graded material (ES-FGM) plate in which FGM consists of two components (ceramic and metal), with in-plane dimensions $a$ and $b$ and thickness $h$ is considered in an orthogonal coordinate system $(O ; x, y, z)$, as shown in Fig. 1. Stiffeners' size $s_{1}, s_{2}$ are the spacing of the longitudinal and transversal stiffeners, respectively; $z_{1}, z_{2}$ are the eccentricities of stiffeners with respect to the middle surface of plate, respectively; $d_{1}, h_{1}$ and $d_{2}, h_{2}$ are the width and thickness of longitudinal and transversal stiffeners, respectively.

\subsection{Material properties of the ES-FGM plates}

The plate is assumed to be made from a mixture of ceramic and metal with the volume-fractions given by a power-law distribution as

$$
V_{m}+V_{c}=1, V_{c}=\left(\frac{2 z+h}{2 h}\right)^{N}
$$

whilst $N$ is the volume fraction exponent and takes only non-negative values, and the subscripts $m$ and $c$ refer to the metal and ceramic constituents, respectively. According to 


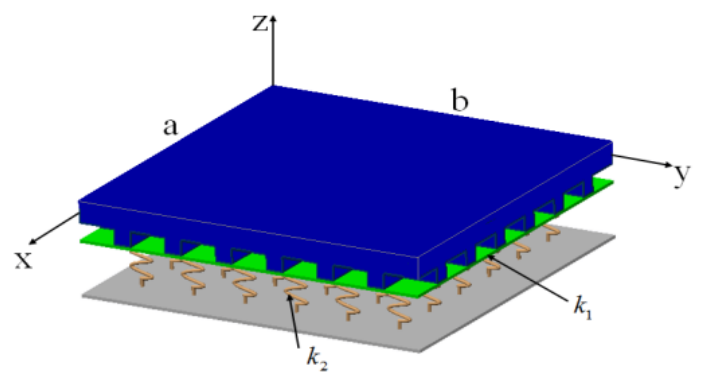

(a)
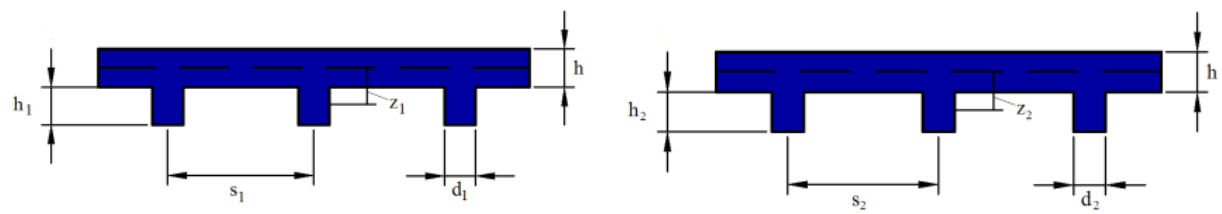

(b)

Fig. 1. (a) Geometry of the ES-FGM plate on elastic foundations;

(b) Geometry of stiffeners of the ES-FGM plate

the mixture rule, the effective properties of the FGM plate can be written as follows

$$
\begin{aligned}
& E(z)=E_{m}+\left(E_{c}-E_{m}\right)\left(\frac{2 z+h}{2 h}\right)^{N}, \quad \alpha(z)=\alpha_{m}+\left(\alpha_{c}-\alpha_{m}\right)\left(\frac{2 z+h}{2 h}\right)^{N}, \\
& \rho(z)=\rho_{m}+\left(\rho_{c}-\rho_{m}\right)\left(\frac{2 z+h}{2 h}\right)^{N}, \quad v=\text { const. }
\end{aligned}
$$

Due to extensive application of the FGM structures in high temperature environments, the material properties of FGM constituents should be considered as a function of temperature. The temperature-dependent typical material property ' $\mathrm{P}$ ' of FGM constituents can be expressed as [7]

$$
P(T)=P_{0}\left(P_{-1} T^{-1}+1+P_{1}^{T}+P_{2} T^{2}+P_{3} T^{3}\right)
$$

whilst $T=T_{0}+\Delta T$ and $T=300 \mathrm{~K}$ (room temperature); $P_{0}, P_{-1} P_{1}, P_{2}$ and $P_{3}$ are temperature-dependent coefficients characterizing the constituent materials.

\section{GOVERNING EQUATIONS FOR THE REDDY'S TSDT MODEL}

\subsection{Displacement field}

Based on Reddy's TSDT [20], the displacement field can be expressed as

$$
u=u^{0}+z \varphi_{x}+z^{3} \frac{4}{3 h^{2}}\left[-\varphi_{x}-\frac{\partial w^{0}}{\partial x}\right], v=v^{0}+z \varphi_{y}+z^{3} \frac{4}{3 h^{2}}\left[-\varphi_{y}-\frac{\partial w^{0}}{\partial y}\right], w=w^{0}
$$


The above displacement fields can be rearranged as proposed by [20]

$$
\begin{gathered}
u=u^{0}-z \frac{\partial w^{0}}{\partial x}+f(z) \phi_{1}^{0}, v=v^{0}-z \frac{\partial w^{0}}{\partial y}+f(z) \phi_{2}^{0}, w=w^{0}, \\
\phi_{1}^{0}=\varphi_{x}+\frac{\partial w^{0}}{\partial x}, \phi_{2}^{0}=\varphi_{y}+\frac{\partial w^{0}}{\partial y} \text { and } f(z)=z\left[1-(4 / 3)(z / h)^{2}\right],
\end{gathered}
$$

here $(u, v, w)$ are displacement components along the $(x, y, z)$ directions, respectively at a distance $z$ away from reference plane and $\left(u^{0}, v^{0}, w^{0}\right)$ are displacement component of a genetic point on the reference surface, $\varphi_{x}$ and $\varphi_{y}$ rotate around the $y$ and $x$ axes, respectively, and the constant $c_{1}=-4 / 3 h^{2}$ in which $h$ is the total thickness of the plate.

\subsection{Strain-displacement relations}

The non-linear strain-displacement relations at a distance ' $z$ ' away from the reference plane of a plate including von Kármán type of nonlinearities are,

$$
\begin{aligned}
& \varepsilon_{x}=\varepsilon_{x}^{0}-z \frac{\partial^{2} w^{0}}{\partial x^{2}}+f(z) \frac{\partial \phi_{1}^{0}}{\partial x}, \varepsilon_{y}=\varepsilon_{y}^{0}-z \frac{\partial^{2} w^{0}}{\partial y^{2}}+f(z) \frac{\partial \phi_{2}^{0}}{\partial y}, \\
& \gamma_{x y}=\gamma_{x x}^{0}-2 z \frac{\partial^{2} w^{0}}{\partial x \partial y}+f(z) \frac{\partial \phi_{1}^{0}}{\partial y}+f(z) \frac{\partial \phi_{2}^{0}}{\partial x}, \\
& \gamma_{x z}=\frac{\partial u^{0}}{\partial z}+\frac{\partial w^{0}}{\partial x}=f^{\prime}(z) \phi_{1}^{0}, \gamma_{y z}=\frac{\partial v^{0}}{\partial z}+\frac{\partial w^{0}}{\partial y}=f^{\prime}(z) \phi_{2}^{0},
\end{aligned}
$$

in which, $\varepsilon_{x}^{0}, \varepsilon_{y}^{0}$ and $\gamma_{x y}^{0}$ are reference surface strains and are defined as

$$
\begin{gathered}
\left.\varepsilon_{x}^{0}=\frac{\partial u^{0}}{\partial x}+\frac{1}{2}\left(\frac{\partial w^{0}}{\partial x}\right)^{2}, \varepsilon_{y}^{0}=\frac{\partial v^{0}}{\partial y}+\frac{1}{2}\left(\frac{\partial w^{0}}{\partial y}\right)^{2}\right], \\
\gamma_{x y}^{0}=\frac{\partial u^{0}}{\partial y}+\frac{\partial v^{0}}{\partial x}+\frac{\partial w^{0}}{\partial x} \frac{\partial w^{0}}{\partial y}, \\
f^{\prime}(z)=\frac{d}{d z} f(z) .
\end{gathered}
$$

Hooke law for an ES-FGM plate under temperature is defined as

$$
\begin{aligned}
& \left(\sigma_{x}, \sigma_{y}\right)=\frac{E}{1-v^{2}}\left[\left(\varepsilon_{x}, \varepsilon_{y}\right)+v\left(\varepsilon_{y}, \varepsilon_{x}\right)-(1+v) \alpha \Delta T(1,1)\right], \\
& \left(\sigma_{x y}, \sigma_{x z}, \sigma_{y z}\right)=\frac{E}{2(1+v)}\left(\gamma_{x y}, \gamma_{x z}, \gamma_{y z}\right),
\end{aligned}
$$

and for stiffeners [5]

$$
\sigma_{x}^{s}=E_{0} \varepsilon_{x}-E_{0} \alpha_{0} \Delta T /\left(1-2 v_{0}\right), \sigma_{y}^{s}=E_{0} \varepsilon_{y}-E_{0} \alpha_{0} \Delta T /\left(1-2 v_{0}\right),
$$

$E_{0}, v_{0}, \alpha_{0}$ are Young's modulus, Poisson ratio and thermal expansion coefficient of the stiffeners, respectively. In order to provide continuity between the plate and the stiffeners, we assume that the stiffeners are made of full metal $\left(E_{0}=E_{m}, \alpha_{0}=\alpha_{m}\right)$ when putting them on the metal-rich side of the plate; and conversely, we assume full ceramic stiffeners $\left(E_{0}=E_{c}, \alpha_{0}=\alpha_{c}\right)$ on the ceramic-rich side of the plate. 
The force and moment resultants of the ES-FGM plate are given in [5]

$$
\begin{aligned}
& \left\{\begin{array}{c}
N_{x} \\
N_{y} \\
N_{x y} \\
M_{x} \\
M_{y} \\
M_{x y} \\
P_{x} \\
P_{y} \\
P_{x y}
\end{array}\right\}=\left[\begin{array}{ccccccccc}
B_{11} & B_{12} & 0 & B_{13} & B_{14} & 0 & B_{15} & B_{16} & 0 \\
B_{12} & B_{22} & 0 & B_{24} & B_{14} & 0 & B_{16} & B_{26} & 0 \\
0 & 0 & B_{31} & 0 & 0 & B_{32} & 0 & 0 & B_{33} \\
B_{13} & B_{14} & 0 & B_{43} & B_{44} & 0 & B_{45} & B_{46} & 0 \\
B_{14} & B_{24} & 0 & B_{44} & B_{54} & 0 & B_{46} & B_{56} & 0 \\
0 & 0 & B_{32} & 0 & 0 & B_{62} & 0 & 0 & B_{63} \\
B_{71} & B_{16} & 0 & B_{73} & B_{46} & 0 & B_{75} & B_{76} & 0 \\
B_{16} & B_{82} & 0 & B_{46} & B_{84} & 0 & B_{76} & B_{86} & 0 \\
0 & 0 & B_{33} & 0 & 0 & B_{63} & 0 & 0 & B_{93}
\end{array}\right]\left\{\begin{array}{c}
\varepsilon_{x}^{0} \\
\varepsilon_{y}^{0} \\
\gamma_{x y}^{0} \\
k_{x}^{1} \\
k_{y}^{1} \\
k_{x y}^{1} \\
k_{x}^{3} \\
k_{y}^{3} \\
k_{x y}^{3}
\end{array}\right\}-\left\{\begin{array}{c}
-B_{17} \varphi_{1}-B_{18} \varphi_{1 x}^{s} \\
-B_{17} \varphi_{1}-B_{28} \varphi_{1 y}^{s} \\
0 \\
-B_{17} \varphi_{2}-B_{18} \varphi_{2 x}^{s} \\
-B_{17} \varphi_{2}-B_{28} \varphi_{2 y}^{s} \\
0 \\
-B_{17} \varphi_{4}-B_{18} \varphi_{4 x}^{s} \\
-B_{17} \varphi_{4}-B_{28} \varphi_{4 y}^{s} \\
0
\end{array}\right\} \\
& \left\{\begin{array}{l}
Q_{x} \\
Q_{y} \\
R_{x} \\
R_{y}
\end{array}\right\}=\left[\begin{array}{cccc}
B_{31} & 0 & B_{62} & 0 \\
0 & B_{31} & 0 & B_{62} \\
B_{62} & 0 & B_{63} & 0 \\
0 & B_{62} & 0 & B_{63}
\end{array}\right]\left\{\begin{array}{l}
\gamma_{x z}^{0} \\
\gamma_{y z}^{0} \\
k_{x z}^{2} \\
k_{y z}^{2}
\end{array}\right\} \\
& \left(\varphi_{1}, \varphi_{2}, \varphi_{4}\right)=\frac{1}{1-v} \int_{-h / 2}^{h / 2}\left(1, z, z^{3}\right) E(z) \alpha(z) \Delta T(z) d z \\
& \left(\varphi_{1 i}^{s}, \varphi_{2 i}^{s}, \varphi_{4 i}^{s}\right)=\frac{1}{1-2 v_{0}} \int_{-h / 2-h_{1}}^{-h / 2}\left(1, z, z^{3}\right) E_{0} \alpha_{0} \Delta T \frac{d_{i}^{T}}{s_{i}^{T}} d z, \quad i=x, y
\end{aligned}
$$

in which, the coefficients are found in the Ref. [5].

After the thermal deformation process, the geometric shapes of stiffeners can be determined as follows [5]

$$
\begin{aligned}
& d_{1}^{T}=d_{1}\left(1+\alpha_{0} \Delta T\right), d_{2}^{T}=d_{2}\left(1+\alpha_{0} \Delta T\right), h_{1}^{T}=h_{1}\left(1+\alpha_{0} \Delta T\right), h_{2}^{T}=h_{2}\left(1+\alpha_{0} \Delta T\right), \\
& s_{1}^{T}=s_{1}\left(1+\alpha_{0} \Delta T\right), s_{2}^{T}=s_{2}\left(1+\alpha_{0} \Delta T\right), z_{1}^{T}=z_{1}\left(1+\alpha_{0} \Delta T\right), z_{2}^{T}=z_{2}\left(1+\alpha_{0} \Delta T\right) .
\end{aligned}
$$

The nonlinear motion equations of an ES-FGM plate on elastic foundations [20]

$$
\begin{aligned}
& N_{x, x}+N_{x y, y}=I_{0} u_{t t}^{0}+J_{1} \varphi_{1, t t}-c_{1} I_{3} w_{x x t}^{0}, \\
& N_{x y, x}+N_{y, y}=I_{0} v_{, t t}^{0}+J_{1} \varphi_{2, t t}-c_{1} I_{3} w_{, y t t}^{0}, \\
& Q_{x, x}+Q_{y, y}-c_{2}\left(R_{x, x}+R_{y, y}\right)+N_{x} w_{, x x}^{0}+2 N_{x y} w_{, x y}^{0}+N_{y} w_{, y y}^{0}+c_{1}\left(P_{x, x x}+2 P_{x y, x y}+P_{y, y y}\right) \\
& -K_{1} w^{0}+K_{2}\left(w_{, x x}^{0}+w_{, y y}^{0}\right)+q(t)=I_{0} w_{, t t}^{0}+2 \varepsilon I_{0} w_{, t}^{0}-c_{1}^{2} I_{6}\left(w_{, x x t t}^{0}+w_{, y y t t}^{0}\right) \\
& +c_{1}\left[I_{3}\left(u_{, x t t}^{0}+v_{, y t t}^{0}\right)+J_{4}\left(\varphi_{x, x t t}+\varphi_{y, y t t}\right)\right], \\
& M_{x, x}+M_{x y, y}-c_{1}\left(P_{x, x}+P_{x y, y}\right)-Q_{x}+c_{2} R_{x}=J_{1} u_{t t}^{0}+K_{2} \varphi_{x, t t}-c_{1} J_{4} w_{x}^{0}, \\
& M_{x y, x}+M_{y, y}-c_{1}\left(P_{x y, x}+P_{y, y}\right)-Q_{y}+c_{2} R_{y}=J_{1} v_{, t t}^{0}+K_{2} \varphi_{y, t t}-c_{1} J_{4} w_{, y t t}^{0},
\end{aligned}
$$


where

$$
\begin{aligned}
& \left(I_{1}, I_{2}, I_{3}, I_{4}, I_{5}, I_{7}\right)=\int_{-h / 2}^{h / 2} \rho(z)\left(1, z, z^{2}, z^{3}, z^{4}, z^{6}\right) d z, \\
& J_{i}=I_{i}-c_{1} I_{i+2}, K_{2}=I_{2}-2 c_{1} I_{4}+c_{1}^{2} I_{6}, c_{2}=3 c_{1},
\end{aligned}
$$

$q(t)$ is blast pressure function, $\varepsilon$ is damping coefficient, $K_{1}$ is Winkler foundation modulus and $K_{2}$ is the shear layer foundation stiffness of Pasternak model.

The well-known Friedlander wave Eq. (15) defines the rise and fall of the static over pressure $P_{s}$ with time as shown by [17]

$$
q(t)=P_{S}(t)=1.8 P S_{\max }\left(1-\frac{t}{T_{S}}\right) \exp ^{-\frac{k t}{T_{s}}},
$$

where the "1.8" factor accounts for the effects of a hemispherical blast, is the maximum (or peak) static over-pressure, is the parameter controlling the rate of wave amplitude decay and is the parameter characterizing the duration of the blast pulse.

The stress function $\psi(x, y, t)$ is introduced as

$$
N_{x}=\psi, y y, N_{y}=\psi, x x, N_{x y}=-\psi, x y .
$$

Replacing Eq. (16) into the two first Eqs. (14) yields

$$
\begin{aligned}
& u_{, t t}^{0}=-J_{1} / I_{0} \varphi_{x, t t}+c_{1} I_{3} / I_{0} w_{, x t t}^{0} \\
& v_{, t t}^{0}=-J_{1} / I_{0} \varphi_{y, t t}+c_{1} I_{3} / I_{0} w_{, y t t}^{0}
\end{aligned}
$$

Substituting Eqs. (8) into Eqs. (11), after substituting expressions of $M_{x}, M_{y}, M_{x y}$, $Q_{x}, Q_{y}$ into Eqs. (14) with the aid of Eqs. (14), we obtain

$$
\begin{aligned}
& L_{11}\left(w^{0}\right)+L_{12}\left(\varphi_{x}\right)+L_{13}\left(\varphi_{y}\right)+L_{14}(\psi)+P\left(w^{0}, \psi\right)=I_{0} w_{, t t}^{0}+2 \varepsilon I_{0} w_{, t}^{0} \\
& +\left(c_{1}^{2} I_{3}^{2} / I_{0}-c_{1}^{2} I_{6}\right)\left(w_{, x x t t}^{0}+w_{, y y t t}^{0}\right)+\left(J_{4} c_{1}-J_{1} I_{3} c_{1} / I_{0}\right) \varphi_{x, x t t}+\left(J_{4} c_{1}-J_{1} I_{3} c_{1} / I_{0}\right) \varphi_{y, y t t}, \\
& L_{21}\left(w^{0}\right)+L_{22}\left(\varphi_{x}\right)+L_{23}\left(\varphi_{y}\right)+L_{24}(\psi)=\left(K_{2}-J_{1}^{2} / I_{0}\right) \varphi_{x, t t}+\left(c_{1} I_{3} J_{1} / I_{0}-c_{1} J_{4}\right) w_{, x t t}^{0} \\
& L_{31}\left(w^{0}\right)+L_{32}\left(\varphi_{x}\right)+L_{33}\left(\varphi_{y}\right)+L_{34}(\psi)=\left(K_{2}-J_{1}^{2} / I_{0}\right) \varphi_{y, t t}+\left(c_{1} I_{3} J_{1} / I_{0}-c_{1} J_{4}\right) w_{, y t t}^{0}
\end{aligned}
$$

in which, the coefficients $L_{i j}$ are found in the Ref. [5].

The system of Eqs. (18) includes four unknown functions $w^{0}, \varphi_{x}, \varphi_{y}$ and $\psi$ so it is necessary to find the fourth equation relating to these functions by using the compatibility equation.

$$
L_{11}\left(\varphi_{x}\right)+L_{12}\left(\varphi_{y}\right)+L_{13}\left(w^{0}\right)+L_{14}(\psi)+P\left(w^{0}, \psi\right)=0,
$$

where $P\left(w^{0}, \psi\right)=\psi, y y w_{, x x}^{0}-2 \psi, x y w_{, x y}^{0}+\psi_{, y y} w_{, y y}^{0}$. 


\section{ANALYTICAL SOLUTIONS}

The simply supported boundary conditions of the ES-FGM plate are [5,20]:

a) Plate edges are simply supported and freely movable (FM). The associated boundary conditions are

$$
\begin{aligned}
& w^{0}=N_{x y}=\varphi_{y}=M_{x}=P_{x}=0, N_{x}=N_{x 0} \text { at } x=0 \text { and } x=a, \\
& w^{0}=N_{x y}=\varphi_{x}=M_{y}=P_{y}=0, N_{y}=N_{y 0} \text { at } y=0 \text { and } y=b .
\end{aligned}
$$

b) The edges are simply supported and immovable (IM). The associated boundary conditions are

$$
\begin{gathered}
w^{0}=u^{0}=\varphi_{y}=M_{x}=P_{x}=0, N_{x}=N_{x 0} \text { at } x=0 \text { and } x=a, \\
w^{0}=v^{0}=\varphi_{x}=M_{y}=P_{y}=0, N_{y}=N_{y 0} \text { at } y=0 \text { and } y=b,
\end{gathered}
$$

in which, $N_{x 0}, N_{y 0}$ are pre-buckling force resultants in directions $x$ and $y$, respectively.

To solve Eqs. (18) and (19) for unknowns $w^{0}, \varphi_{x}, \varphi_{y}$ and $\psi$, and with the consideration of the boundary conditions (20)-(21), we assume the following approximate solutions

$$
\begin{aligned}
& w^{0}=W(t) \sin \alpha x \sin \beta y, \varphi_{x}=\varphi_{x}(t) \cos \alpha x \sin \beta y, \varphi_{y}=\varphi_{y}(t) \sin \alpha x \cos \beta y, \\
& \psi=A_{1} \cos 2 \alpha x+A_{2} \cos 2 \beta y+A_{3} \sin \alpha x \sin \beta y+N_{x 0} y^{2} / 2+N_{y 0} x^{2} / 2 .
\end{aligned}
$$

Therein, $\alpha=\frac{m \pi}{a}, \beta=\frac{n \pi}{b}, m, n=1,2, \ldots$ are numbers of half waves in $x, y$ directions, respectively, and $W$ is amplitude of deflection. Also, $A_{i}(i=1-3)$ are coefficients to be determined.

After substituting Eqs. (22) into Eq. (19), the coefficients $A_{i}(i=1-3)$ are found

$$
A_{1}=W^{2} \frac{\beta^{2} /}{\alpha^{2}} \frac{\Delta}{32 B_{11}}, A_{2}=W^{2} \frac{\alpha^{2}}{\beta^{2}} \frac{\Delta}{32 B_{22}}, A_{3}=\frac{H_{2}}{H_{1}} \varphi_{x}+\frac{H_{3}}{H_{1}} \varphi_{y}+\frac{H_{4}}{H_{1}} W .
$$

Introduction of Eqs. (22) into Eqs. (18), and applying the Galerkin method for the resulting equation yields

$$
\begin{aligned}
l_{11} W+l_{12} \varphi_{x} & +l_{13} \varphi_{y}+l_{14} \varphi_{x} W+l_{15} \varphi_{y} W+l_{16} W^{2}+l_{17} W^{2}-\left(N_{x 0} \alpha^{2}+N_{y 0} \beta^{2}\right) W \\
& +16 q / m n \pi^{2}=n_{2} W_{, t t}+2 \varepsilon I_{0} W_{, t}+\rho_{2} m \pi / a \varphi_{x, t t}+\rho_{2} n \pi / b \varphi_{y, t t}, \\
l_{21} W+l_{22} \varphi_{x} & +l_{23} \varphi_{y}+l_{24} W^{2}=\rho_{1} \varphi_{x, t t}+\rho_{2} m \pi / a W_{, t t}, \\
l_{31} W+l_{32} \varphi_{x} & +l_{33} \varphi_{y}+l_{34} W^{2}=\rho_{1} \varphi_{y, t t}+\rho_{2} n \pi / b W_{, t t},
\end{aligned}
$$

$m, n$ are odd numbers and $\rho_{1}=K_{2}-J_{1}^{2} / I_{0}, \rho_{2}=c_{1} I_{3} J_{1} / I_{0}-c_{1} J_{4}$, specific expressions of coefficients $l_{i j}(i=1 \div 3, j=1 \div 3), n_{1}, n_{2}$ are found in the Ref. [5].

Considering the ES-FGM plate with all edges which are simply supported and immovable under thermal load, the condition expressing the immovability on the edges, $u^{0}=0$ (on $\left.x=0, a\right)$ and $v^{0}=0($ on $y=0, b)$, is satisfied in an average sense as $[5,20]$

$$
\int_{0}^{b} \int_{0}^{a} \frac{\partial u^{0}}{\partial x} d x d y=0, \int_{0}^{a} \int_{0}^{b} \frac{\partial v^{0}}{\partial y} d x d y=0
$$


From Eqs. (6), (12) and (20), we obtain the following expressions

$$
\begin{aligned}
u_{, x}^{0}= & B_{22} / \Delta \psi_{, y y}-B_{12} / \Delta \psi_{, x x}+b_{11} \varphi_{y, y}+b_{12} \varphi_{x, x}+b_{13} w_{, x x}^{0} \\
& +b_{14} w_{, y y}^{0}-b_{14} \varphi_{1}-B_{28} B_{12} / \Delta \varphi_{1 y}+B_{18} B_{22} / \Delta \varphi_{1 x}-\left(w_{, x}^{0}\right)^{2} / 2, \\
v_{, y}^{0}= & B_{11} / \Delta \psi, x x-B_{12} / \Delta \psi_{, y y}+b_{21} \varphi_{x, x}+b_{22} \varphi_{y, y}+b_{23} w_{, x x}^{0} \\
& +b_{24} w_{, y y}^{0}-b_{25} \varphi_{1}-B_{18} B_{12} / \Delta \varphi_{1 x}+B_{11} B_{28} / \Delta \varphi_{1 y}-\left(w_{, y}^{0}\right)^{2} / 2 .
\end{aligned}
$$

Putting Eqs. (22) into Eq. (25) then substituting the obtained result into Eqs. (24), we have

$$
\begin{aligned}
& N_{x 0}=e_{11} W+e_{12} \varphi_{x}+e_{13} \varphi_{y}+e_{14} W^{2}+e_{15} \varphi_{1}-e_{16} \varphi_{1 x}, \\
& N_{y 0}=e_{21} W+e_{22} \varphi_{x}+e_{23} \varphi_{y}+e_{24} W^{2}+e_{25} \varphi_{1}-e_{26} \varphi_{1 y} .
\end{aligned}
$$

Replacing Eqs. (26a), and (26b) into the equations of motion (23), we have

$$
\begin{aligned}
& {\left[l_{11}-e_{15} \alpha^{2} \varphi_{1}-e_{25} \beta^{2} \varphi_{1}+e_{16} \alpha^{2} \varphi_{1 x}+e_{26} \beta^{2} \varphi_{1 y}\right] W+l_{12} \varphi_{x}+l_{13} \varphi_{y}+l_{14} \varphi_{x} W+l_{15} \varphi_{y} W} \\
& \quad+l_{16} W^{2}+l_{17} W^{3}+16 /\left(m n \pi^{2}\right) q=n_{2} W_{, t t}+2 \varepsilon I_{0} W_{, t}+\rho_{2} m \pi / a \varphi_{x, t t}+\rho_{2} n \pi / b \varphi_{y, t t}, \\
& l_{21} W+l_{22} \varphi_{x}+l_{23} \varphi_{y}=\rho_{1} \varphi_{x, t t}+\rho_{2} m \pi / a W_{, t t}, \\
& l_{31} W+l_{32} \varphi_{x}+l_{33} \varphi_{y}=\rho_{1} \varphi_{y, t t}+\rho_{2} n \pi / b W_{, t t} .
\end{aligned}
$$

Taking linear parts of the set of Eqs. (27) and putting $q=0$, the natural frequencies $(\omega)$ of the plate can be determined directly by solving determinant

$$
\left|\begin{array}{ccc}
l_{11}-e_{15} \alpha^{2} \varphi_{1}-e_{25} \beta^{2} \varphi_{1} & l_{12}+\rho_{2} \frac{m \pi}{a} \omega^{2} & l_{13}+\rho_{2} \frac{n \pi}{b} \omega^{2} \\
+e_{16} \alpha^{2} \varphi_{1 x}+e_{26} \beta^{2} \varphi_{1 y}+n_{2} \omega^{2} & l_{22}+\rho_{1} \omega^{2} & l_{23} \\
l_{21}+\rho_{2} \alpha \omega^{2} & l_{32} & l_{33}+\rho_{1} \omega^{2}
\end{array}\right|=0 .
$$

Solving Eq. (28) yields three angular frequencies of the ES FGM plate, and the smallest one is being considered.

The plate is placed in the environment whose temperature is steadily increased from the beginning value $T_{i}$ to the last value $T_{f}$, the temperature difference $\Delta T=T_{f}-T_{i}$ is a constant.

$\varphi_{a}$ is determined,

$$
\varphi_{a}=P \Delta T ; \quad P=h\left(E_{m} \alpha_{m}+\left(E_{m} \alpha_{c m}+E_{c m} \alpha_{m}\right) /(N+1)+E_{c m} \alpha_{c m} /(2 N+1)\right) .
$$

\section{NUMERICAL EXAMPLES AND DISCUSSION}

In this section, the components of the material are silicon nitride $\mathrm{Si}_{3} \mathrm{~N}_{4}$ (ceramic) and SUS304 stainless steel (metal). The material properties $P_{r}$ in the formula Eq. (3) are shown in Ref. [5,7], and a Poisson's ratio $v=0.3$ is chosen for simplicity.

We have assumed that the stiffeners are made of full metal, so $E_{0}=E_{m}$. The parameters for the stiffeners are: $h_{1}=h_{2}=0.08(\mathrm{~m}), s_{1}=s_{2}=0.15(\mathrm{~m}), d_{1}=d_{2}=0.008(\mathrm{~m})$, $E_{0}=E_{m}, \alpha_{0}=\alpha_{m}$. 


\subsection{Numerical verification}

In particular case of a FGM plate without stiffeners under the conditions: $A_{1}=A_{2}=$ 0 , we have compared the numerical results of un-stiffened FGM plate with Ungbhakorn and Wattanasakulpong [8]. They used the energy function and choosing results under the type of moving position function for study and without eccentrically stiffener. From the Tab. 1, it can be seen that the present values are not significantly different from the result in [8].

Table 1. Comparison of fundamental frequency parameter $\gamma=\omega h \sqrt{\frac{\rho_{c}}{E_{c}}}$ for $\mathrm{Al} / \mathrm{Al}_{2} \mathrm{O}_{3}$ square plates without elastic foundations and $(a / b=1,(m, n)=(1,1), \Delta T=0)$

\begin{tabular}{|c|c|c|c|c|c|c|}
\hline \multirow{2}{*}{ Source } & \multicolumn{3}{|c|}{$a=10 h$} & \multicolumn{3}{c|}{$a=5 h$} \\
\cline { 2 - 7 } & $N=0.5$ & $N=1.0$ & $N=10.0$ & $N=0.5$ & $N=1.0$ & $N=10.0$ \\
\hline Ref. [8] & 0.0490 & 0.0442 & 0.0364 & 0.1807 & 0.1631 & 0.1301 \\
\hline Present & 0.05 & 0.0440 & 0.0369 & 0.1829 & 0.1640 & 0.1300 \\
\hline
\end{tabular}

\subsection{Natural frequencies}

The effects of elastic foundations on the natural frequency of the eccentrically stiffened plate are shown in Tab. 2. Increasing $K_{1}$ leads to increasing the natural frequencies of the ES FGM plates. The Tab. 2 also shows that the lowest natural frequency corresponds mode $(m, n)=(1,1)$.

Table 2. Effect of the elastic foundations, stiffener and buckling mode $(m, n)$ on natural frequencies of the ES-FGM plates

\begin{tabular}{|c|c|c|c|c|c|c|c|c|}
\hline & \multicolumn{2}{|c|}{$\omega_{1}$} & \multicolumn{2}{c|}{$\begin{array}{c}\omega_{2} \\
(m=1, n=1)\end{array}$} & \multicolumn{2}{c|}{$\begin{array}{c}\omega_{3} \\
(m=1, n=3)\end{array}$} & \multicolumn{2}{c|}{$\begin{array}{c}\omega_{4} \\
(m=3, n=5)\end{array}$} \\
\hline $\begin{array}{c}K_{1} \\
(\mathrm{GPa} / \mathrm{m})\end{array}$ & Stiffener & Unstiffener & Stiffener & Unstiffener & Stiffener & Unstiffener & Stiffener & Unstiffener \\
\hline 0 & 28800 & 2278 & 43805 & 11126 & 81230 & 27723 & 108278 & 35552 \\
\hline 0.1 & 28811 & 2409 & 43812 & 11153 & 81232 & 27734 & 108281 & 35561 \\
\hline 0.3 & 28832 & 2651 & 43826 & 11207 & 81240 & 27756 & 108287 & 35576 \\
\hline 0.35 & 28838 & 2708 & 43831 & 11220 & 81243 & 27761 & 108288 & 35580 \\
\hline 0.5 & 28854 & 2872 & 43841 & 11261 & 81248 & 27777 & 108292 & 35593 \\
\hline 0.7 & 28875 & 3079 & 43854 & 11316 & 81255 & 27798 & 108298 & 35611 \\
\hline
\end{tabular}

\subsection{Nonlinear dynamic response}

The nonlinear dynamic response of stiffened and un-stiffened FGM plate is analyzed in Fig. 2. It is obvious that the result is a support for dynamic response and vibration of 


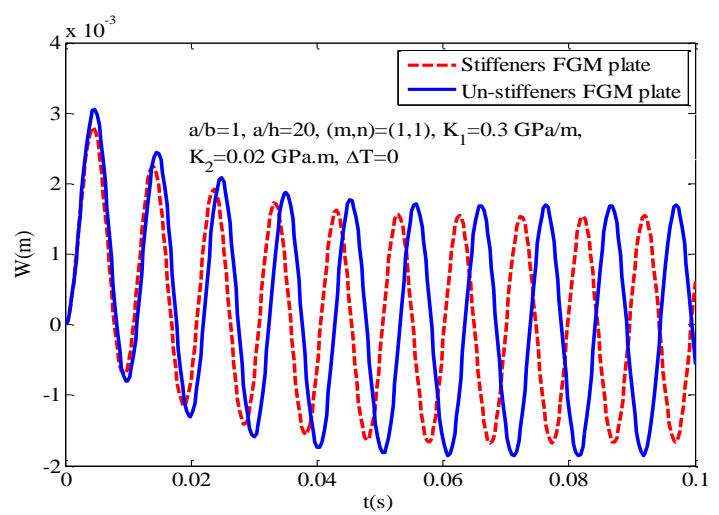

Fig. 2. Effect of stiffeners on nonlinear dynamic response of the ES-FGM plates under blast load

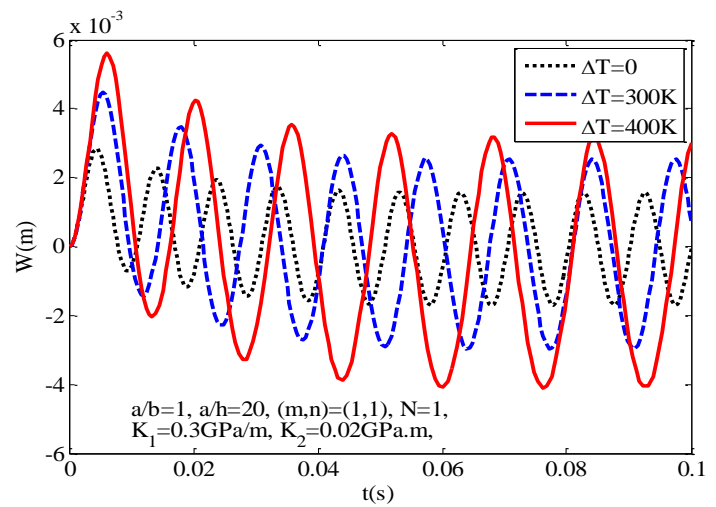

Fig. 3. Effect of temperature on nonlinear response of the ES-FGM plates under blast load

ES-FGM plate. In another word, the vibration amplitude of the FGM plate is strongly decreased by the stiffeners.

The effect of temperatures $\Delta T(K)=(0,300,400)$ on the nonlinear dynamic response of the ES-FGM plates is shown in Fig. 3. It can be seen that the dynamic response amplitude increases when the temperature $\Delta T$ increases. It means that the dynamic response amplitude is in direct proportion to the temperature $\Delta T$.

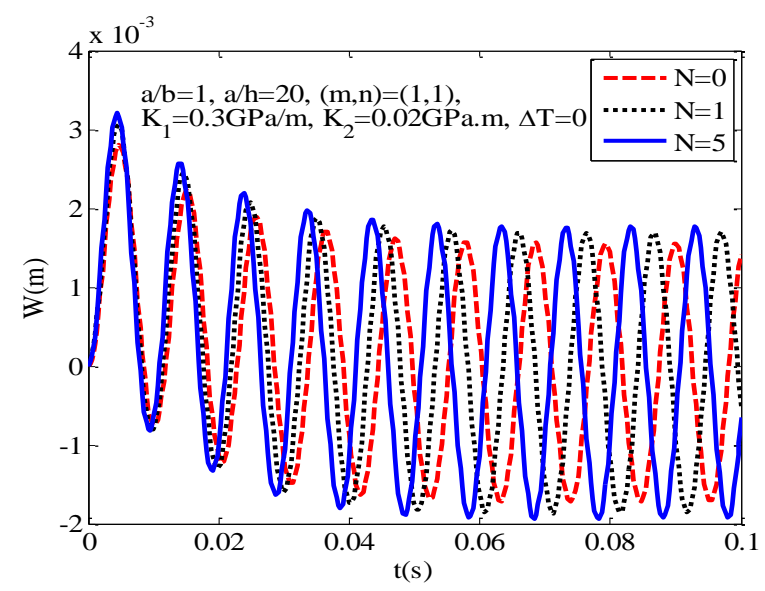

Fig. 4. Effect of power law index $N$ on nonlinear dynamic response of the ES-FGM plates under blast load

Fig. 4 shows the effect of the power law index $N$ on the nonlinear dynamic response of the ES-FGM plates with $a / b=1, a / h=20$ of the ES-FGM plate with $N=0,1,5$. Obviously, the amplitude of the nonlinear dynamic response of the ES-FGM plate is directly proportional to the power law index $N$. 
Figs. 5 and 6 illustrate the effect of geometric factors of the ES-FGM plates on nonlinear dynamic response with $N=1$. From Fig. 5, it can be seen that the amplitude of the ES FGM plates increases when increasing the ratio $a / b$. Fig. 6 shows the effect of the ratio $b / h$ on the nonlinear dynamic response of the ES-FGM plates. When increasing the ratio $b / h$, the plate fluctuates more strongly.

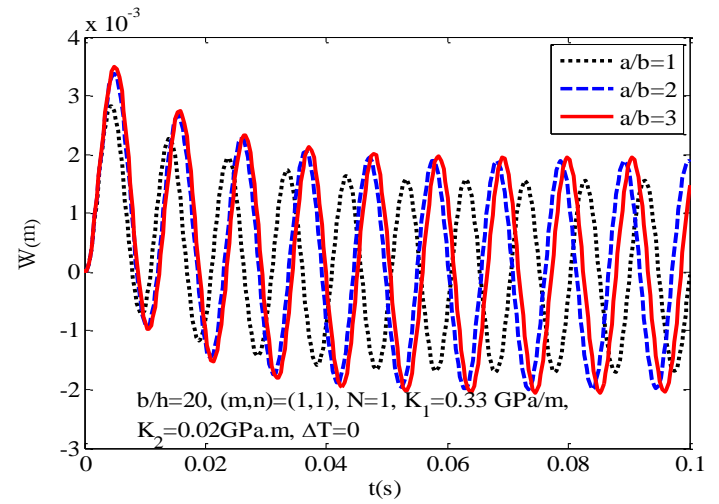

Fig. 5. Effect of ratio $a / b$ on nonlinear dynamic response of the ES-FGM plates under blast load

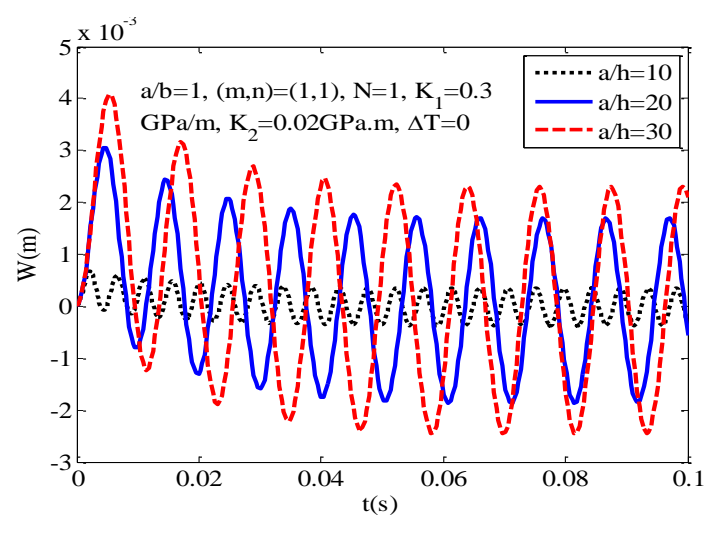

Fig. 6. Effect of ratio $a / h$ on nonlinear dynamic response of the ES-FGM plates under blast load

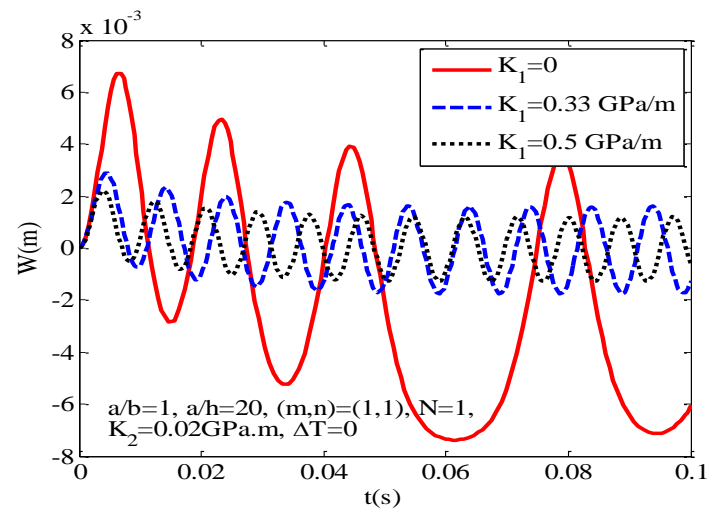

Fig. 7. Effect of the linear Winkler foundation on nonlinear dynamic response of the ES-FGM plates under blast load

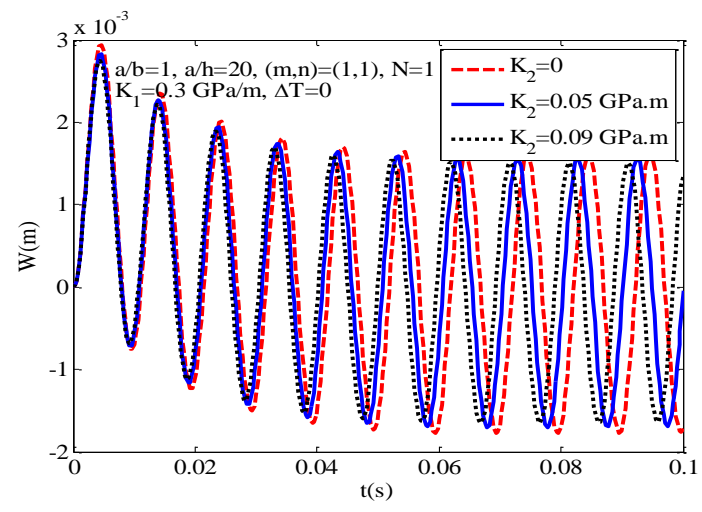

Fig. 8. Effect of the Pasternak foundation on nonlinear dynamic response of the ES-FGM plates under blast load

Figs. 7 and 8 show the effect of elastic foundations on the nonlinear dynamic response of the ES-FGM plates with $a / b=1, a / h=20, N=1$. Fig. 7 presents the effect of the Winkler foundation. It is clear that the plate fluctuation amplitude decreases only when the module $K_{1}$ of Winkler foundation increases. In conclusion, the plate fluctuation amplitude is in inverse proportion to the module $K_{1}$ of Winkler foundation. The parameter $K_{2}$ of the Pasternak foundation also has similar behavior. The graphs in Figs. 7 and 8 illustrate the beneficial effects of elastic foundations on the nonlinear dynamic response 
Pham Hong Cong, Nguyen Dinh Duc

of the ES-FGM plates. The amplitude of the plate decreases when it is rested on elastic foundations.

\section{CONCLUDING REMARKS}

In this paper, the nonlinear dynamic of the ES-FGM under blast and thermal loads in thermal environment are studied and discussed. Based on Reddy's TSDT, stress function, Galerkin method and fourth order Runge-Kutta method, some general inferences are mentioned as below:

- The stiffener system strongly enhances the load-carrying capacity of the ES-FGM plates.

- The elastic foundations and temperature have strong effect on the nonlinear dynamic response of the ES-FGM plates, and the dynamic response amplitude of the plate increases when the temperature increases.

- Geometrical parameters $(a / b, a / h$ ratios) and the volume ratio $N$ have extremely impacted on the nonlinear dynamic responses of the ES-FGM plates.

\section{ACKNOWLEDGEMENT}

This research is funded by Vietnam National Foundation for Science and Technology Development (NAFOSTED) under grant number 107.02-2015.03. The authors are grateful for this support.

\section{REFERENCES}

[1] N. D. Duc, P. H. Cong, and V. D. Quang. Nonlinear dynamic and vibration analysis of piezoelectric eccentrically stiffened FGM plates in thermal environment. International Journal of Mechanical Sciences, 115, (2016), pp. 711-722. doi:10.1016/j.ijmecsci.2016.07.010.

[2] D. V. Dung and N. T. Nga. Nonlinear analysis of stability for imperfect eccentrically stiffened FGM plates under mechanical and thermal loads based on FSDT. Part 1: Governing equations establishment. Vietnam Journal of Mechanics, 37, (3), (2015), pp. 187-204. doi:10.15625/0866-7136/37/3/5884.

[3] N. D. Duc. Nonlinear static and dynamic stability of functionally graded plates and shells. Vietnam National University Press, Hanoi, (2014).

[4] N. D. Duc, P. H. Cong, N. D. Tuan, P. Tran, V. M. Anh, and V. D. Quang. Nonlinear vibration and dynamic response of imperfect eccentrically stiffened shear deformable sandwich plate with functionally graded material in thermal environment. Journal of Sandwich Structures $\mathcal{E}$ Materials, 18, (4), (2016), pp. 445-473. doi:10.1177/1099636215602142.

[5] P. H. Cong, V. M. Anh, and N. D. Duc. Nonlinear dynamic response of eccentrically stiffened FGM plate using Reddys TSDT in thermal environment. Journal of Thermal Stresses, 40, (6), (2017), pp. 704-732. doi:10.1080/01495739.2016.1261614.

[6] F. Alijani and M. Amabili. Non-linear dynamic instability of functionally graded plates in thermal environments. International Journal of Non-Linear Mechanics, 50, (2013), pp. 109-126. doi:10.1016/j.ijnonlinmec.2012.10.009.

[7] J. N. Reddy and C. D. Chin. Thermomechanical analysis of functionally graded cylinders and plates. Journal of Thermal Stresses, 21, (6), (1998), pp. 593-626. doi:10.1080/01495739808956165. 
[8] V. Ungbhakorn and N. Wattanasakulpong. Thermo-elastic vibration analysis of third-order shear deformable functionally graded plates with distributed patch mass under thermal environment. Applied Acoustics, 74, (9), (2013), pp. 1045-1059. doi:10.1016/j.apacoust.2013.03.010.

[9] P. T. Thang, N. D. Duc, and T. Nguyen-Thoi. Thermomechanical buckling and post-buckling of cylindrical shell with functionally graded coatings and reinforced by stringers. Aerospace Science and Technology, 66, (2017), pp. 392-401. doi:10.1016/j.ast.2017.03.023.

[10] D. V. Dung and V. H. Nam. Nonlinear dynamic analysis of eccentrically stiffened functionally graded circular cylindrical thin shells under external pressure and surrounded by an elastic medium. European Journal of Mechanics-A/Solids, 46, (2014), pp. 42-53. doi:10.1016/j.euromechsol.2014.02.008.

[11] N. D. Duc, V. D. Quang, and V. T. T. Anh. The nonlinear dynamic and vibration of the S-FGM shallow spherical shells resting on an elastic foundations including temperature effects. International Journal of Mechanical Sciences, 123, (2017), pp. 54-63. doi:10.1016/j.ijmecsci.2017.01.043.

[12] D. H. Bich, D. V. Dung, and V. H. Nam. Nonlinear dynamic analysis of eccentrically stiffened imperfect functionally graded doubly curved thin shallow shells. Composite Structures, 96, (2013), pp. 384-395. doi:10.1016/j.compstruct.2012.10.009.

[13] N. D. Duc. Nonlinear dynamic response of imperfect eccentrically stiffened FGM double curved shallow shells on elastic foundation. Composite Structures, 99, (2013), pp. 88-96. doi:10.1016/j.compstruct.2012.11.017.

[14] D. H. Bich, N. D. Duc, and T. Q. Quan. Nonlinear vibration of imperfect eccentrically stiffened functionally graded double curved shallow shells resting on elastic foundation using the first order shear deformation theory. International Journal of Mechanical Sciences, 80, (2014), pp. 16-28. doi:10.1016/j.ijmecsci.2013.12.009.

[15] D. T. Hau, N. T. M. Hanh, and N. T. Trung. A cell-based smoothed discrete shear gap method (CS-FEM-DSG3) for dynamic response of laminated composite plate subjected to blast loading. Vietnam Journal of Mechanics, 37, (2), (2015), pp. 81-90. doi:10.15625/08667136/37/2/5019.

[16] N. D. Duc and P. H. Cong. Nonlinear dynamic response and vibration of sandwich composite plates with negative Poissons ratio in auxetic honeycombs. Journal of Sandwich Structures $\mathcal{E}$ Materials, (2016). doi:10.1177/1099636216674729.

[17] N. Lam, P. Mendis, and T. Ngo. Response spectrum solutions for blast loading. Electronic Journal of Structural Engineering, 4, (4), (2004), pp. 28-44.

[18] N. D. Duc, N. D. Tuan, P. Tran, and T. Q. Quan. Nonlinear dynamic response and vibration of imperfect shear deformable functionally graded plates subjected to blast and thermal loads. Mechanics of Advanced Materials and Structures, 24, (4), (2017), pp. 318-329. doi:10.1080/15376494.2016.1142024.

[19] N. T. Duong and N. D. Duc. Evaluation of elastic properties and thermal expansion coefficient of composites reinforced by randomly distributed spherical particles with negative Poissons ratio. Composite Structures, 153, (2016), pp. 569-577. doi:10.1016/j.compstruct.2016.06.069.

[20] J. N. Reddy. Mechanics of laminated composite plates and shells: Theory and analysis. CRC Press, (2004). 